\title{
“ONCO-AESTETHIC”, A new medical approach to improve quality of life in cancer patients
}

\author{
Alexander Bianchi ${ }^{1}$, Bianca Maria De Cesaris ${ }^{2}$, Alfonso Baldi ${ }^{1,3 *}$ and Andrea Garelli ${ }^{1}$ \\ ${ }^{1}$ Master in Aestethic Medicine, Fondazione Policlinico Tor Vergata, Tor Vergata University, Rome, Italy \\ ${ }^{2}$ Università Cattolica del Sacro Cuore; Fondazione Policlinico Universitario A. Gemelli IRCCS, Rome, Italy \\ ${ }^{3}$ Department of Environmental, Biological, Pharmaceutical Sciences and Technologies, University of Campania "L. Vanvitelli”, Caserta, Italy
}

\section{Communication}

Health-related quality of life is now considered a significant endpoint in studies of outcomes in oncology. Indeed, quality of life valuations in cancer patients may contribute to enhanced treatment and could even be of prognostic value [1]. Nevertheless, the quality of life of people affected by cancer is still neglected: doctors rarely address this aspect, few patients talk about it, and in a great number of the studies still their "well-being" is excluded from the criteria for evaluating the effectiveness of cancer therapy. Aesthetics and cancer are two concepts that are kept separate by instinct. It seems almost disrespectful to even imagine that a woman, or even more so a man, who has been diagnosed with cancer still has the desire and time to feel beautiful. But it is a mistake, especially since we too often underestimate how important the psychological aspect and relationships are in a therapeutic process. Early pivotal studies, lasting more than 30 years ago, showed already a positive relationship between some psychosocial and quality of life parameters and survival time in cancer patients [2,3]. Currently, a great number of studies have clearly demonstrated that global quality of life or global health status is associated with prolonged survival time [4].

Chemotherapy is fundamental in the treatment of cancer: medical research has led to therapies that significantly extend the patient's life expectancy but remains a very demanding and debilitating treatment. Therefore, there is a need for collateral therapies that take care of the patient's life. Recognizing yourself in the mirror, taking care of your image, doing body care treatments, having interests and leisure are all fundamental elements to keep your social life intact and gain a state of well-being every day.

An exemplificative case is the skin toxicity of anti-cancer therapies (dryness, itching, burning, for example). This condition negatively affects the psychophysical well-being of people, also increasing the emotional distress (distress) which increases the risk of interrupting cancer therapy. For this reason, specific aesthetic treatments and cosmetic products during and after cancer therapy, for the management of skin reactions, should be included in the treatment protocols because psychophysical well-being favours adherence to therapy and the effectiveness of treatments in the fight against cancer. This is particularly true in breast cancer patients. A very recent study from the University of Milan and the European Institute of Oncology with a research that involved 170 women in breast cancer therapy, followed by applying the protocols of the beauticians of the Association oncological aesthetics professional (APEO), demonstrated that adequate aesthetic treatments can improve the quality of life of people undergoing cancer therapy by halving stress [5].
In our experience, the treatment of a cancer patient needs a multidisciplinary group made up by pathologists, clinicians, plastic surgeons, oncologists, aesthetic doctors, but also by psychologists, beauty professionals including aestheticians, wig makers, make-up experts. Together they guide the cancer patient towards recovery.

We have proposed the term of "onco-aesthetic" to define this is new branch of medicine, a form of "oncological aesthetics" whose purpose is to improve the psychophysical well-being of cancer patients thanks to a set of activities that accompany them in the treatment and sometimes therapeutic process. A project that arises from the need to improve the attitude towards psychophysical care of cancer patients. The concept can be summarized in a motto: "the patient must live, not survive!"

Searching online for the definition of "well-being", the first result mentions: "Harmonic state of health, physical and spiritual strength". Too often, the announcement of cancer by the doctor is not proposed in the right way, in fact there is a tendency to address the patient as if the tumour is the only aspect of one's life to look after. So many leave their jobs, others lock themselves up in a state of depression, still others turn away from their affections. What is lacking in the healthcare system and what the onco-aesthetic project is committed to disseminating is attention to the patient's quality of life. Preparing him for the consequences of chemotherapy and the changing body means not only warning but also proposing a solution.

Among the side effects of chemotherapy there is alopecia, but also paleness, weight gain and water retention, in some cases disfiguring skin rashes. Aesthetic medicine, make-up, psychological support, alternative medicine, and much more are disciplines that together help the patient and accompany him on the path of recovery from cancer with awareness and self-love. It has been shown that the effectiveness of a therapy increases in the presence of a positive psychophysical state [4]. The perception of one's dignity as a human being must be cultivated in every moment.

"Being valued as women at a time when you don't feel beautiful helps morally" Angela, a cancer patient treated by our team, is one of the voices, indeed, of the smiles of "onco-aesthetics". She works in

${ }^{*}$ Correspondence to: Alfonso Baldi, Department of Environmental, Biological, Pharmaceutical Sciences and Technologies, University of Campania "L. Vanvitelli", Caserta, Italy, E-mail: alfonsobaldi@tiscali.it

Received: September 07, 2020; Accepted: September 14, 2020; Published: September 17, 2020 
the world of entertainment; she is an actress. From her experience she says she was able to continue the theatrical tour even though they had anticipated that she would lose her hair. Like many other women, she has had a wig since the beginning of therapy. Chemotherapy without hair loss is unfortunately not possible but cutting hair before it falls out on its own is important to avoid trauma.

Our expectation is that soon this all-round approach to cancer patients will become more and more applied. This, together with sound methodological approaches and adequate statistical analyses will likely yield accurate and specific quality of life-related prognostic variables for specific cancers and adequate multidisciplinary approaches to improve prognosis of these patients.

\section{References}

1. Coons SJ (2007) Health-related quality of life: let's measure and report it appropriately. Clin Ther 29: 2746-2747.

2. Cassileth BR, Lusk EJ, Miller DS, Brown LL, Miller C (1985) Psychosocial correlates of survival in advanced malignant disease?. N Engl J Med 312: 1551-1555.

3. Coates A, Gebski V, Bishop JF, Jeal PN, Woods RL, et al. (1987) Improving the quality of life during chemotherapy for advanced breast cancer. A comparison of intermittent and continuous treatment strategy. N Engl J Med 317: 1490-1495.

4. Montazeri A (2009) Quality of life data as prognostic indicators of survival in cancer patients: an overview of the literature from 1982 to 2008. Health Qual Life Outcomes 7: 102

5. Oliveri S, Faccio F, Pizzoli S, Monzani D, Redaelli C, et al. (2019) A pilot study on aesthetic treatments performed by qualified aesthetic practitioners: efficacy on healthrelated quality of life in breast cancer patients. Qual Life Res 28: 1543-1553.

Copyright: (C2020 Bianchi A. This is an open-access article distributed under the terms of the Creative Commons Attribution License, which permits unrestricted use, distribution, and reproduction in any medium, provided the original author and source are credited. 